\title{
Erratum to: Desultory Topics in Quantum Mechanics
}

E. L. Michelsen, Quirky Quantum Concepts, Undergraduate Lecture Notes in Physics, DOI 10.1007/978-1-4614-9305-1_8, ( ) Springer Science+Business Media New York 2014

The original version of Chapter 8 was inadvertently published with errors. Those errors have been corrected as follows:

Page 324. "8.3.3 Raising and Lowering: How Did Dirac Do It?" should read as follows.

\subsubsection{Raising and Lowering: How Did Dirac Do It?}

The "operator method" of harmonic oscillator analysis is fundamental to all advanced QM. It foreshadows a very similar method used for angular momentum, and is the basis of a major part of QFT (including quantum EM radiation), which starts with the fields as an infinite set of quantized harmonic oscillators. The method is far from obvious, so the big question on everybody's mind is "How did Dirac do it?" How did he know how to create the creation and annihilation operators? Most references give them as "Lo! And behold!" (much like they present the Schrödinger equation out of thin air). Here is one way to do it logically, and (who knows?) perhaps is similar to how Dirac figured it out. Our goal here is not to provide the simplest derivation, but to show how such an idea might come about in the first place. In addition, this section further ties together the meaning of operators and Dirac notation, which makes this topic worth understanding. This section assumes you understand how the operator method works, but not how to motivate its development.

First, recall the stationary states of the 1D harmonic oscillator ([8], 7.18, p. 144):

$$
\begin{aligned}
|n\rangle \equiv u_{n}(x) & =\frac{1}{\sqrt{2^{n} n !}}\left(\frac{m \omega}{\hbar \pi}\right)^{1 / 4} H_{n}\left(\sqrt{\frac{m \omega}{\hbar}} x\right) \exp \left(-\frac{m \omega}{2 \hbar} x^{2}\right) \\
& =C_{n} H_{n}(X) \exp \left(-\frac{1}{2} X^{2}\right)
\end{aligned}
$$

The updated original online version of this chapter can be found at https://doi.org/10.1007/978-1-4614-9305-1_8 


$$
\begin{aligned}
\text { where } X & \equiv \sqrt{\frac{m \omega}{\hbar}} x \text { is dimensionless position, } C_{n} \equiv \text { normalization. } \\
H_{n}(X) & \equiv \text { Hermite polynomial of degree } n
\end{aligned}
$$

Now, consider two recursion relations between the Hermite polynomials, (which imply relations between the harmonic oscillator stationary states), which were well known long before Dirac ([7], 7.19-20, p. 144):

$$
\begin{aligned}
\frac{d}{d X} H_{n}(X) & =2 n H_{n-1}(X), \\
\text { and } \quad 2 X H_{n}(X) & =H_{n+1}(X)+2 n H_{n-1}(X) .
\end{aligned}
$$

In the second equation, we already see the seeds of the well-known relation $\hat{x} \propto\left(\hat{a}^{\dagger}+\hat{a}\right)$, because it says [after multiplying through by $\left.\exp \left(-X^{2} / 2\right)\right]$ :

$$
\begin{aligned}
& \hat{x}|n\rangle \propto b|n+1\rangle+c|n-1\rangle, \\
& \text { where } b, c \text { are as-yet unknown numbers. }
\end{aligned}
$$

Also, the first recursion relation tells us something about momentum operators, since $\hat{p} \propto d / d x:$

Now Dirac is famous for "Dirac notation," which is a coordinate-free (i.e., representation-indepent) notation for quantum states, and other kets. We seek some purely conceptual relations between the state kets, which must be independent of representation. Therefore we ask: can we write the Hermite recursion relations in ket notation? We start with the first step: can we write a Hermite polynomial in ket notation? Sure:

$$
u_{n}(x)=C_{n} H_{n}(\cdot) \exp (\cdot) \Rightarrow H_{n}(x)=\frac{1}{C_{n}} \exp \left(+X^{2} / 2\right) \underset{|n\rangle}{u_{n}(x)}=\frac{1}{C_{n}} \exp \left(+X^{2} / 2\right)|n\rangle \text {. }
$$

Since the first recursion relation in Eq. (8.7) includes a derivative, what is the derivative of a Hermite polynomial in ket notation? We simply differentiate the above equation:

$$
\frac{d}{d X} H_{n}(X)=\frac{d}{d X} \frac{1}{C_{n}} \exp \left(+X^{2} / 2\right)|n\rangle .
$$

In the $x$-representation, $|n\rangle$ is a function of $x$, so we use the product rule on the RHS:

$$
\begin{aligned}
\frac{d}{d X} H_{n}(X) & =\frac{1}{C_{n}}\left\{\left[\frac{d}{d X} \exp \left(+X^{2} / 2\right)\right]|n\rangle+\exp \left(+X^{2} / 2\right) \frac{d}{d X}|n\rangle\right\} \\
& =\frac{1}{C_{n}}\left\{\left[X \exp \left(+X^{2} / 2\right)\right]|n\rangle+\exp \left(+X^{2} / 2\right) \frac{d}{d X}|n\rangle\right\} \\
& =\frac{1}{C_{n}} \exp \left(+X^{2} / 2\right)\left\{X+\frac{d}{d X}\right\}|n\rangle .
\end{aligned}
$$


These results show that the first recursion relation relates $|n\rangle$ to $|n-1\rangle$; when written in ket notation, this gives us a lowering operator. After canceling the $\exp \left(+X^{2} / 2\right)$ :

$$
\frac{1}{C_{n}}\left\{X+\frac{d}{d X}\right\}|n\rangle=2 n \frac{1}{C_{n-1}}|n-1\rangle \text {. }
$$

We eliminate the normalization factors from:

$$
C_{n} \propto 1 / \sqrt{2^{n} n !} \Rightarrow C_{n} / C_{n-1}=1 / \sqrt{2 n} .
$$

We now return to the true position variable. Use:

$$
X=\sqrt{\frac{m \omega}{\hbar}} x, \quad \frac{d}{d X}=\sqrt{\frac{\hbar}{m \omega}} \frac{d}{d x}=\sqrt{\frac{\hbar}{m \omega}} \frac{i}{\hbar} \hat{p} .
$$

To be representation-indpendent, we also take $x \rightarrow \hat{x}$. Then Eq. (8.9) becomes:

$$
\left\{\sqrt{\frac{m \omega}{\hbar}} \hat{x}+i \sqrt{\frac{1}{\hbar m \omega}} \hat{p}\right\}|n\rangle=\sqrt{2 n}|n-1\rangle \text { or }\left\{\sqrt{\frac{m \omega}{2 \hbar}} \hat{x}+i \sqrt{\frac{1}{2 \hbar m \omega}} \hat{p}\right\}|n\rangle=\sqrt{n}|n-1\rangle \text {. }
$$

This gives the lowering operator in final form:

$$
\hat{a}|n\rangle=\sqrt{n}|n-1\rangle \text { where } \hat{a}=\sqrt{\frac{m \omega}{2 \hbar}} \hat{x}+i \sqrt{\frac{1}{2 \hbar m \omega}} \hat{p} .
$$

As shown later, this is sufficient to also define a raising operator, which is the adjoint of $\hat{a}$. However, to continue with our wave-function derivation, we first find the raising operator directly from the recursion relations Eq. (8.7). For a raising operator, we write them with $H_{n+1}$ on the left, and only $H_{n}$ on the right:

$$
H_{n+1}(X)=2 X H_{n}(X)-\frac{d}{d X} H_{n}(X) .
$$

We already know how to convert this to ket notation. After again canceling all the $\exp \left(+X^{2} / 2\right)$ :

$$
\begin{aligned}
\frac{1}{C_{n+1}}|n+1\rangle & =\frac{2}{C_{n}} \sqrt{\frac{m \omega}{\hbar}} \hat{x}|n\rangle-\frac{1}{C_{n}}\left\{\sqrt{\frac{m \omega}{\hbar}} \hat{x}+i \sqrt{\frac{1}{\hbar m \omega}} \hat{p}\right\}|n\rangle \\
& =\frac{1}{C_{n}}\left\{\sqrt{\frac{m \omega}{\hbar}} \hat{x}-i \sqrt{\frac{1}{\hbar m \omega}} \hat{p}\right\}|n\rangle \quad \text { Use: } C_{n} / C_{n+1}=\sqrt{2(n+1)} \\
\sqrt{n+1}|n+1\rangle & =\left\{\sqrt{\frac{m \omega}{2 \hbar}} \hat{x}-i \sqrt{\frac{1}{2 \hbar m \omega}} \hat{p}\right\}|n\rangle \Rightarrow \hat{a}^{\dagger}=\sqrt{\frac{m \omega}{2 \hbar}} \hat{x}-i \sqrt{\frac{1}{2 \hbar m \omega}} \hat{p} .
\end{aligned}
$$

We see explicitly that the raising operator is the adjoint of the lowering operator.

Algebraic derivation of $\hat{\boldsymbol{a}}^{\dagger}$ : As noted ealier, we can derive the raising operator as the adjoint of the lowering operator more simply by using Dirac algebra. Start with Eq. (8.10), and use the definition of adjoint: 


$$
\hat{a}|n\rangle=\sqrt{n}|n-1\rangle \Rightarrow \quad\langle n| \hat{a}^{\dagger}=\sqrt{n}\langle n-1| .
$$

Since $n$ is arbitrary, we can replace $n \rightarrow n+1$ :

$$
\begin{aligned}
\langle n+1| \hat{a}^{\dagger} & =\sqrt{n+1}\langle n| \Rightarrow\left\langle n+1\left|\hat{a}^{\dagger}\right| n\right\rangle=\sqrt{n+1} \text {, and }\left\langle m\left|\hat{a}^{\dagger}\right| n\right\rangle=0, m \neq n+1 \\
& \Rightarrow \quad \hat{a}^{\dagger}|n\rangle=\sqrt{n+1}|n+1\rangle .
\end{aligned}
$$

A key property of $\hat{a}$ and $\hat{a}^{\dagger}$ is that $\hat{x}$ and $\hat{p}$ are not just linear combinations of them, but proportional to their sum and difference:

$$
\hat{x} \propto\left(\hat{a}+\hat{a}^{\dagger}\right), \quad \hat{p} \propto\left(\hat{a}-\hat{a}^{\dagger}\right) .
$$

This greatly simplifies computing inner products of $\hat{x}$ and $\hat{p}$. Also, note that $\hat{a}$ and $\hat{a}^{\dagger}$ are real; the appearance of $i$ with $\hat{p}$ in Eqs. (8.10) and (8.11) cancels the $i$ in the definition $\hat{p} \equiv(\hbar / i) \nabla$.

Of course now that we know these results, we could rederive all this much more quickly. That requires a "lo and behold" approach, where we show that a seemingly random definition of the operator $\hat{a}$ leads to a useful result. Many references supply such a derivation. Our goal here, though, was to show how one might develop this result from observations about well-known prior results. 